\title{
Access to Opioids in Palliative Care in Low-and Middle-Income Countries: The Case of Burkina-Faso How Can Blockchain and Internet of Things Assist?
} \section{Type of article: Editorial}

\author{
Thierry Edoh ${ }^{1}$, Martin Lankoande ${ }^{2}$ \\ 1 Department of Pharmacy, University of Bonn, Germany \\ 2 Teaching Hospital Yalgado Ouédroago, in Burkina Faso.
}

\begin{abstract}
Background: People requesting palliative care or suffering from pain are subjected to adhere to opioidbased treatment in order to alleviate their pain. Commonly, access to opioids is strictly controlled. Access to Healthcare delivery services remains challenging in Low-and Middle-Income Countries (LMIC). In Burkina-Faso (BF), a Sub-Saharan African (SSA) country, patients requiring palliative care (PC) are especially facing poor access to pain drugs such as morphine. Facing poor access to pain-alleviating medicine can severely impact the daily quality of life $(\mathrm{QoL})$. On one hand, patients are experiencing poor opioids access. On another hand opioids abuse, leading to drug addiction is noticed. The question arising here is how can they face poor access and at the same time abuse the given drug? One plausible answer is the counterfeit chain, which provides illegal drugs. Beyond the counterfeit issues faced, the public health care system is also facing, amongst others, prescription falsification, fraud in the distribution, and stock shortage.
\end{abstract}

Objectives: The main objectives are to fight against opioid addiction, counterfeits, a stock shortage, and prevent related health safety issues. The main aim is to enable the traceability of any opioids prescription, secure the supply and distribution, and thus early detect any fraud in the system.

This editorial paper would, therefore, focus on investigating the reasons underlying the poor access to opioids in palliative care in BF and make suggestions for improvement. A blockchain (BC) and the Internet of Things (IoT) based system to secure and improve opioids supply, distribution, and prescription will be proposed.

Method \& Design: Mixed-Method-Design was applied to this study. National stipulations, regulations, and the state-of-the-art in the field of palliative care in BF were investigated and analyzed. Based on the investigation's outcomes and following the paradigm of design science research, and information system based improvement solution is proposed to tackle the poor access to opioids, improve the supply and distribution chain as well as to efficiently monitor the consumption of opioids in $\mathrm{BF}$, and prevent patients from any health issues, drug addiction, and death.

Results: The contribution analysis reveals the potential of the proposed model to assist in many ways to improve access to opioids and to secure this access. The model could contribute to preventing drug abuse, overprescription, supporting off-label-use of opioids and thus providing a knowledge database for off-label use of opioids. This model shows promise to deliver accurate data and information about the exact opioid's needs and consumption atlas. This will assist to better distribute the product in the entire country.

A proof-of-concept of the proposed model is required. This is ongoing and will be presented in a forthcoming paper.

Conclusion: This editorial paper investigates access to opioids in Burkina Faso. It pointed out by analyzing out the computer science perspectives the different causes of the crisis. A contextualized model is provided. A test in situ needs to be performed.

Keywords: Poor Access to Healthcare, Drug Supply Chain, Drug Distribution, Palliative Care, Internet of Things for Healthcare, Blockchain for Healthcare, Quality of Life, Morphine Provision.

Corresponding author: Thierry Edoh RFW-Universität Bonn, Bonn Germany email: oscar.edoh@ gmail.com

Screened by iThenticate..@2017-2019 KNOWLEDGE KINGDOM PUBLISHING.

\section{Introduction}

Morphine is one of the widespread pain-drug after Tramadol that patients requiring palliative and/or pain care get prescribed by their medical doctors. Frequently in-taking and misusing opioid analgesic substances present a severe risk of addiction and can lead 
Medical Technologies Journal, Volume: 3, Issue: 3, July-September 2019, Pages: 430-442. Doi : https://doi.org/10.26415/2572-004X-vol3iss3p430-442

to death. Precisely for these reasons, prescribing this class of drug is challenging for medical doctors (MD), especially for those who are not trained for palliative and pain care like anesthesiologists. Though, the global health care system is facing the illegal drug market with all related issues. Catherine Monfajon, a French journalist, has investigated the misuse of tramadol (opioid) in Africa. She reports the outcomes of her investigations ARTE, a European TV channel. The report is available at https://www.arte.tv/en/videos/088982-000-A/togo-s-tramadol-problem/ (Available from $30 / 09 / 2019$ to $27 / 08 / 2022$ ). The results of this work reveal to what extent tramadol, a painkiller, is misused and becomes in entire Africa a major public health care problem. Burkina Faso (BF) is also facing this scourge. Painkillers are easily accessible at the "black market" and people prefer purchasing them there for simple reasons like (i) high price of the drug at the legal pharmacy and (ii) lack statutory health insurance [1]. Beyond issues related to counterfeits of opioids, available at black markets, many public health care systems worldwide also face overprescribing of opioids [2- 4], prescription forgery. African countries, especially, face additionally stock-shortage issues. Disciplinary actions are taken against physicians who overprescribe opioids [5]. Healthcare authorities, worldwide, therefore, strictly control and monitor the supply and distribution of opioids. The intention of the healthcare authorities is to prevent any health safety issues that patients can face by abusively using opioid analgesic substances. As a consequence, access to opioids is restrictive. However, the restrictive access to opioid analgesics like morphine leads or can drive to poor access to palliative and pain care, especially in Lowand Middle-Income Countries (LMIC).

When analyzing the proliferation of opioids at the African black market, it is worth noticing that only the politic can sustainably stem this scourge. The main question arising is to know where these products come from.

Unlike purchasing fake or counterfeit drugs at the black market in developing countries, the developed countries face the import of counterfeits through the Internet. In this case, technology can help to trace any drug purchase on the Internet.

High-Income Countries (HIC) dispose of excellent pharmaceutical supply chain and distribution channels (like high modern pharmacies and modern drug transport logistics). There also exist legal and regulatory stipulations governing the distribution or sale of medicine, especially opioid analgesic substances. In Germany, for example, only a few MD can prescribe opioid analgesic substances since they are classified as a narcotic drug. The prescribing of these substances is restrictive and strictly regulated. For traceability and health safety purposes, MDs are under the duty to use a special prescription form (Gelber Arztrezept, eng. yellow prescription form) to prescribe such drugs. Each form is registered with the health authorities, has a serial number, and is a bundle of three strains. The MD, who has prescribed the substance, keeps a strain the pharmacist also keeps one strain. The last strain is sent to the healthcare authorities. Each prescription must be reported to the health authorities. All these legal dispositions demonstrate how dangerous the said substances could be. The main objectives that healthcare authorities are pursuing in this case (Germany) are to prevent narcotic drugs addicted to forge prescription or misuse and, thus, prevent them from health safety issues.

While high-income countries (HIC) have put effort to prevent any misuse of opioid analgesic substances as well as trace the provenance of opioid analgesic prescription and thus, ensure access to palliative and/or pain care (P\&PC), LMIC are facing various challenges issues regarding the opioid analgesic accessibility. Purchasing pharmaceutical 
Medical Technologies Journal, Volume: 3, Issue: 3, July-September 2019, Pages: 430-442. Doi :

https://doi.org/10.26415/2572-004X-vol3iss3p430-442

products in LMIC is commonly challenging since most pharmacies are facing stockshortages for multiple reasons Stock shortage, price of the medicine, cheap counterfeits available at the black market, and lack of health insurance to cover health-related costs are the leading causes of poor access to pharmaceutical care in LMIC. Beyond these causes, poor supply chain and distribution systems, as well as lack of possibility to trace the prescription provenance, are additional causes of poor access to opioid analgesic substances such as morphine in LMIC. Therefore, the first research question is What are the leading causes of morphine stock shortage in BF? In BF, the pharmacies are provided with opioids by the government. No pharmacy can, at its own, order abroad opioids. Though, this aims to investigate Why the government is supplying a low stock of morphine?

Nowadays, modern technologies are used to improve health and pharmaceutical care accessibility in HIC as well in LMIC [1], [6]- [8]. and to collect data [9]. Since modern technology has shown promise to assist to overcome challenges and issues in certain fields of healthcare, it, therefore, judicious to develop an information system model using modern information and communication technology (ICT) for improving the opioid supply and distribution chains with the main objective to prevent any drug abuse as well as prevent the population against health safety issues. This model should include the traceability of prescriptions from their genesis to medicine purchasing. The research question arising here is Can modern information and communication technology assist in solving the morphine accessibility issues and challenges faced? What are these challenges and issues? Furthermore, this study foresees to investigate how to use the technology to prevent health care professionals including pharmacists against legal liability. Though, the study's relevant question is How to secure the morphine prescription and trace the provenance in order to prevent healthcare professionals such as pharmacists and medical doctors to face any legal complaint? The objective of the case study on morphine accessibility challenges and issues in BF, which is reported in this paper is to provide a better understanding of the issues causing the poor morphine accessibility in Burkina-Faso (BF) and to propose innovation solution approaches using modern information technology. The main focus of this study is on design, implementing, and deploying an information technology-enabled poor access solution rather than palliative and pain care delivery and why medical doctors decline to prescribe opioid analgesic substances. This study just focuses on ensuring prescription provenance.

This research seeks to answer the main research questions and verify the hypotheses.

\section{Problem Statement and Analysis}

The analysis of the opioid supply and distribution process (supply and distribution chains) at the legal "drug market" in Burkina Faso, the prescription and the purchase at a pharmacy, reveals that the government controls the provision of most products from abroad and many different health bodies monitor the distribution and collect information on the distribution and consumption of legal drugs. This procedure is similar in all developing countries which lack pharmaceutical industries and must purchase abroad. In Africa, especially in BF our study case, the ministry of health $(\mathrm{MoH})$ oversees coordinating, purchasing opioids abroad. Prior to the order abroad, many structures of the $\mathrm{MoH}$, like DGPML, select and determine the number of opioids to be ordered. The 
Medical Technologies Journal, Volume: 3, Issue: 3, July-September 2019, Pages: 430-442. Doi :

https://doi.org/10.26415/2572-004X-vol3iss3p430-442

structures of the $\mathrm{MoH}$ are also in charge of supplying the purchased opioids to legal pharmacies in the entire country.

The opioid regulations and stipulations request pharmacies to report the movement of the opioids as well as the consumption. For these purposes, paper-based report forms have been prepared [10]. Paper-based reports are limited and can bear errors since the information containing is transcribed by human beings. Furthermore, the stipulations request the pharmacists to lock the product in available deposit boxes and, thus, prevent any inappropriate access to the product.

The health care system further faces prescription forgery problems. Many addicted people use that way to legally buy opioids and misuse it. Medical doctors overprescribe these medicaments for two reasons (i) they have no control over the patient records [Medical Record issues], thus, they are not really aware of being overprescribing. (ii) Provide a better quality of life to the patient in prescribing the product off the label what results in overprescription and off-label-use of the given product.

With respect to above-discussed issues and analyzing the whole out the perspective of computer science, a question is, therefore, arises to what extent can the Information System assist to overcome the given issues in order to:

1.Improve access to opioids?

2.Ensure health safety and prevent any opioid abuse and addiction?

3.Secure the drug stock against frauds?

4.Assist medical doctors in prescribing and thus avoid or prevent overprescription and off-label prescriptions?

5.Build a knowledge database for off-label use of opioids?

6.Improve information collection on opioids consumption and thus precisely determine the needs of opioids in the whole country?

7.Assist the $\mathrm{MoH}$ and affiliated department to gain accurate information?

\section{Blockchain and Internet of Things loT based Contextualized Model}

The main issues, when the problem is analyzed in the viewpoint of computer science, are on one hand the information gathering methodology and the support used for this purpose. On the other hand, the BF public health care system like most African care systems lacks electronic medical records (EMR). The problem analysis points out the association between the lacking EMR/EHR and overprescribing.

This section presents a contextualized model to overcome the issues facing.

\section{Drug Supply chain, Distribution and Consumption Report}

BF MoH provides some directives [10], [11] on how to monitor the distribution of opioids in the entire and how to determine the number of opioids that the BF health care system needs. Purposely, the different department of the $\mathrm{MoH}$, hospitals, clinics, as well as the pharmacies, are being provided with paper-based forms to report the consumption by inpatients, opioids purchase at pharmacies, the quarterly countrywide consumption report. A Blockchain-enabled report form is proposed to improve and ensure the different reports. Blockchain technology is combined with the Internet of things (IoT) technology (Figure 
Medical Technologies Journal, Volume: 3, Issue: 3, July-September 2019, Pages: 430-442. Doi : https://doi.org/10.26415/2572-004X-vol3iss3p430-442

1) for this purpose. Blockchain (BL) can assist to secure the system entries (data and information) so that no change can happen during the transfer from one station to the other (e. g. from a pharmacy to local government service and vice versa). No one can change any information without informing the other partners on the network. Data consistency and integrity are though guaranteed. It will be easy to detect fraud and who commit it (the essence of blockchain). Each actor on the blockchain network owns a copy of each transaction (reports on opioids, etc.). BL can, therefore, assume system robustness and prevent against cyberattacks, assure data integrity [12]. Since Blockchain fundamentally opts for transparency where transactions are cryptographically signed but unencrypted, sensible medical data needs additional technology to encrypt the data so that only authorized persons can access the information containing in the data.

Today, there exists various blockchain-enabled health platform to better secure health generated data. In [13], the authors present healthbank, a blockchain-based platform, for securing health data. Beyond the security and privacy aspect, Blockchain technology, a distributed ledger technology (DLT), relies on secured distributed database technology, which history is traceable [14]. BL can be used for an efficient supply chain process. It assists to reduce transaction costs and time. It improves visibility across the supply chain and thus offers increased transparency since the entries (ledgers) are open for any trading partner [15]. The government and the pharmacies are trading partners, where the government is the supplier in this context. BL Technology would, therefore, provide more transparency, connect government institutions to the pharmacies for better control and monitoring of opioid consumption, prevent fraud, and would provide accurate information on the real opioid needs or demand.

IoT technology has the potential to judiciously autonomously and automatically collect data dependently on the system settings. IoT is using for many health applications such as patient remote monitoring [16]. Originally, IoT uses RFID (Radio Frequency Identification) technology to identify any movement an object is making [17]. Today, many perception units like sensors are used to catch data that are transmitted over the IoT platform to a remote server (e. g. often in the cloud). Using this technology to monitor will assist to seamlessly collect accurate data on opioid consumption, distribution in the entire country. The collected data will assist the government to accurately to determine the opioid's demand and needs countrywide and, thus, it could order the required number of opioids. Ordering the exact required number of opioids will partially resolve the opioid shortage since the new cases could be predicted by the system. Based on this, a prediction system is needed on top so that the government can forecast supplement orders of opioids to face new palliative cases and related opioid demand.

As a Study assumption

Combining both BL and IoT could assist, on one side to accurately collect information from pharmacies, hospitals, and clinics. On the other side, this will resolve the opioid shortage (H1).

Figure 1 shows the architecture of a blockchain for the internet of things. Collected data are automatically stored in the distributed database underlying the blockchain part of the system. The traditional data ingestion layer of an IoT architecture is though replaced with the data ingestion layer of the blockchain. The docked blockchain architecture includes two technologies to assure data interoperability by data exchanges within the blockchain network. Both technologies are (i) HL7 assist to format medical data and (ii) FHIR assure 
Medical Technologies Journal, Volume: 3, Issue: 3, July-September 2019, Pages: 430-442. Doi :

https://doi.org/10.26415/2572-004X-vol3iss3p430-442

secure and interoperable data exchanges. A smart contract processor assists in computing smart contracts between the different nodes on the network.

\subsection{Combating Drug Abuse and Counterfeits}

Overprescription of legal opioids, unaware off-label use of opioids, sale of counterfeits available at the black market are direct causes of opioid abuse that can lead to health safety issues as well as death.

Despite numerous efforts that the government is putting together to stem the counterfeit scourge in the entire country, many addicted purchase opioids at the black markets, even palliative patients also purchase opioids at the black market for diverse reasons such as high prices at the legal pharmacies, a stock shortage, addiction, intensive pain, etc.

The proposed model foresees a social media-like platform for monitoring and collecting data about drug abuse. Parents, relatives, or friends can anonymously report cases of drug abuse. Any addicted can report on himself and ask for help. Beyond the monitoring platform, video-based visual monitoring systems must be used at places like schools, universities, and other public places. This video-based monitoring should work like the system proposed in [18] to monitor flooding events. Using video sensing presents many advantages amongst others, the dissuasive effect of the presence of the video. Combining both video sense, included in the IoT system, and the social platform will assist to reduce the extent of illegal intake of and deal with opioids at any public place. Though, counseling works need to be done with young addicted people to show them the negative impact of illegally intaking opioids.

Combating counterfeits using information systems in the context of Africa, and particularly in BF would not work because consumers (patients and addicted, health bodies and others) are aware that the products sold at the market are counterfeits, though they purchase them due to the high prices practiced at the legal pharmacies. A prominent approach here will be to launch health insurance that will cover medical expenses.

Unlike counterfeits available at the market, commonly and wrongly called "Generic drug", some counterfeit drugs are being sold in pharmacies. Though, since 2015 a verification system called MPedigree is used to fight against counterfeits drugs in Africa. However, MPedigree presents some limitations since only the drug's containers or packages are labeled. One can replace the package's contents with counterfeits and retail the legal drugs in detail since selling detail is often practiced in Africa. The drug's packages need to be sealed and can only be open with a unique key that can only be tried once.

Blockchain technology can also be applied to secure drugs against fraud. This topic will be discussed and a model will be presented in the forthcoming paper.

\subsection{Improve Off-Label Prescribing and Use of Opioids}

Off-label prescribing and use is a worldwide phenomenon. Many countries own stipulations and regulations regarding prescribing and using a medicine off label. In Germany, off-label is legally allowed but strict regulated, while this is strictly forbidden in the USA.

Off-label prescription and use mostly rely on long-term experiences of medical doctors. Therefore, the proposed model features large and decentral/distributed knowledge 
Medical Technologies Journal, Volume: 3, Issue: 3, July-September 2019, Pages: 430-442. Doi : https://doi.org/10.26415/2572-004X-vol3iss3p430-442

database using the blockchain technology to secure the entries again falsification but keep a high level of transparency so that any health care professionals can benefit from experiences of others (experiences exchanges with same requirements as data exchanges).

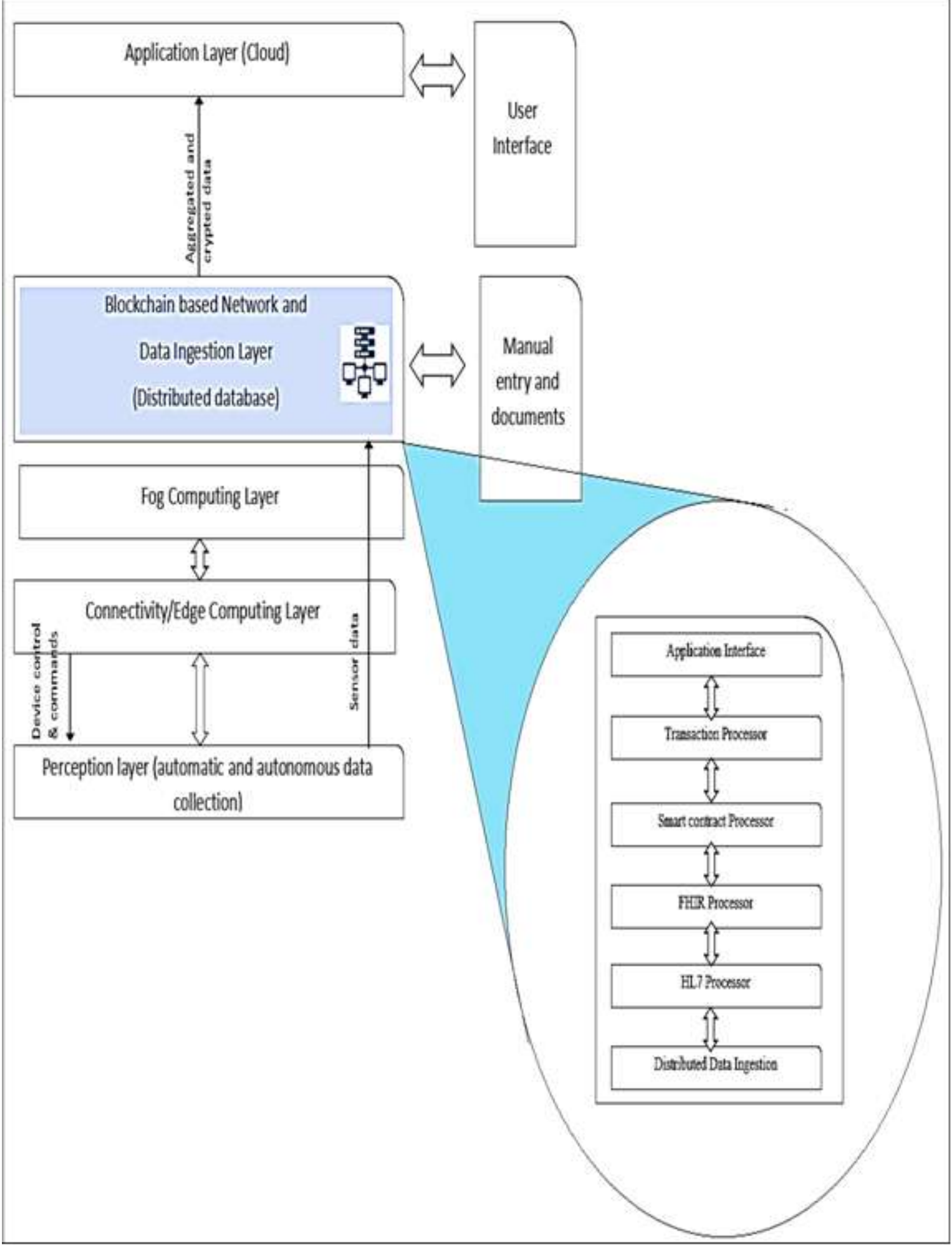

Figure 1: Architecture Palliative Care related data and documents exchange system architecture (using Blockchain for the Internet of Things) 
Medical Technologies Journal, Volume: 3, Issue: 3, July-September 2019, Pages: 430-442. Doi :

https://doi.org/10.26415/2572-004X-vol3iss3p430-442

\subsection{Overprescribing}

The further problem the BF public health care, as all other public health care systems, opioid abuses. Patients or people can access certain opioids like tramadol at the black market. Unlike tramadol, morphine is difficult to be purchased at the black market. In order to control the morphine intake, the $\mathrm{MoH}$ released several directives like monitor exactly who dispenses the products, who consumes it. These details have to be provided to the government. Though, many people forge prescriptions and can thus "legally" and easily purchase the product at a pharmacy. This model has answered the question of how technology can assist here to prevent prescription forgery, overprescribing, and opioid abuse.

To avoid overprescribing, medical doctors (MD) need more information on patient medication. However, such information is often recorded in a booklet kept by the patient at home. Such booklets are lost and the containing too [19]. Depth analysis of the overprescribing issues reveals that the lack of medical and health data following by offlabel use of the products is the leading cause of overprescribing. Regarding the leading causes, a disturbed and ubiquitous electronic health / medical records (EMR/HER) system is needed to centralize the patient-data. These data would be accessible anywhere and anytime so that the patient can visit any MD they want.

EMR systems as required here should be robust against any cyberattack in order to prevent any data integrity issue. Combining blockchain technology with HL7/FHIR (Health Level 7/ Fast Healthcare Interoperability Resources) presents two advantages

(i) data integrity [20]and (ii) interoperable data exchange supported by HL7/FHIR standards. FHIR uses external cryptography to enable data privacy and integrity. The model foresees to uses the FHIR, standards to enable data interoperability, on the blockchain technology to distribute and secure the data. In [21], the authors implemented a blockchain-supported EMR that enable the data interoperability and secure the data. However, their proposed solution does not use the FHIR standard. HL7 and FHIR are the widely used standard in the medical field to feature data interoperability. EMR carries important data that can impact patient health outcomes [22].

Additionally, to Blockchain technology, the IoT can also contribute to collect key data that could be integrated into EMR.

The second assumption is that

EMR can contribute to reducing overprescribing and stemming prescription forgery. (H2)

However, the model is limited to fight drug abuse supported by black-market opioids. Figure 2 presents at a glance the architectural view of a prescription supporting system. The medical doctor is connected to the patient medical records, lying in the central database. The data exchange between the medical doctor and the medical records system is supported by the blockchain technology in order to assure data integrity during the transaction. Any prescription is forwarded to the blockchain network comprising the $\mathrm{MoH}$, health bodies, patients, pharmacies, and any relevant actor. The system automatically provides the patient with a copy of the prescription that he will use to purchase the given drug. 
Medical Technologies Journal, Volume: 3, Issue: 3, July-September 2019, Pages: 430-442. Doi : https://doi.org/10.26415/2572-004X-vol3iss3p430-442

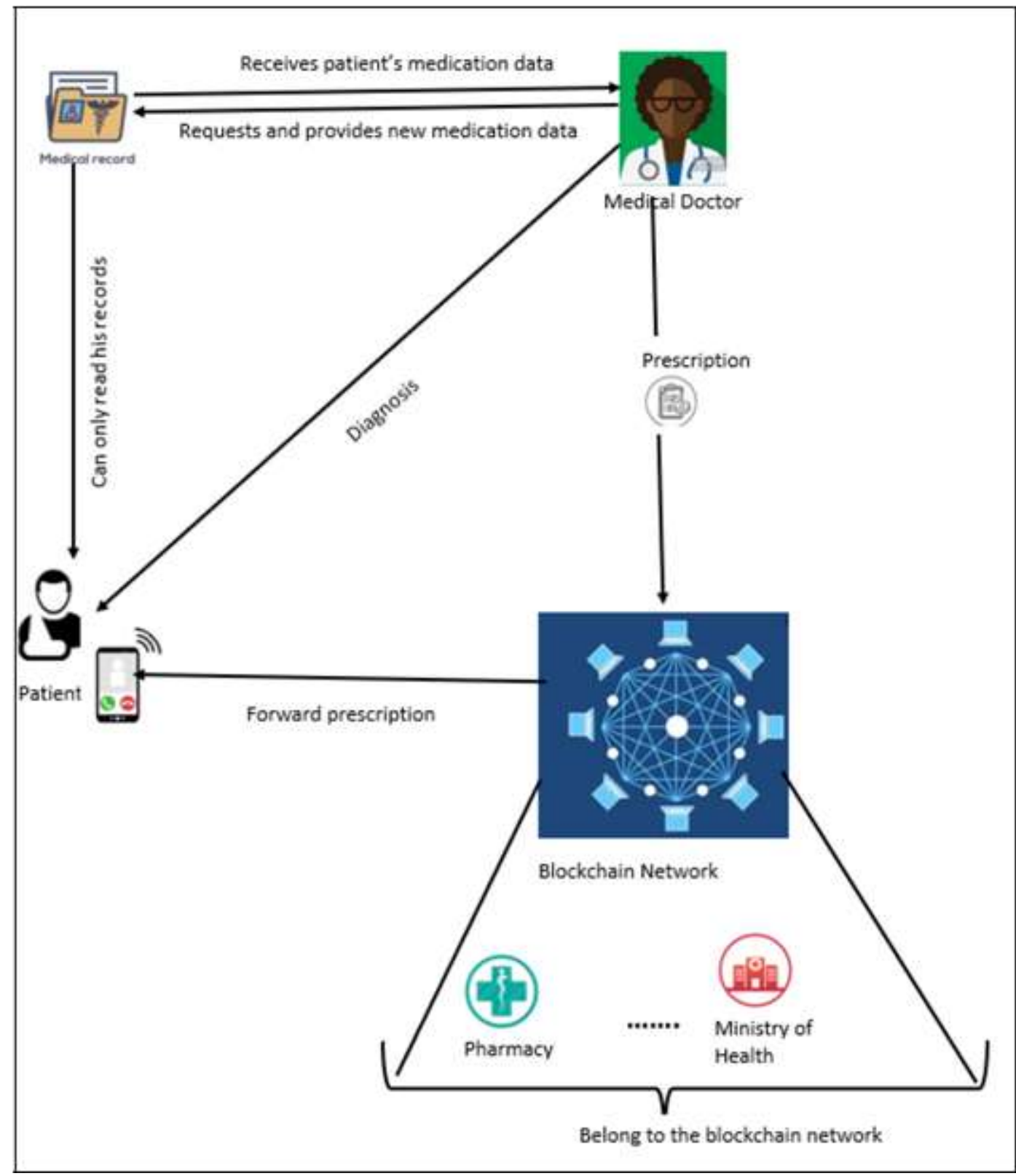

Figure 2: Prescription Process (Preventing any overprescription)

\section{Contribution Analysis}

This section analyzes the contribution of this editorial study to the literature as well as to the improvement of palliative care in BF. Two assumptions ( $\mathrm{H} 0$ \& $\mathrm{H} 1)$ were made and need to be verified.

\subsection{Research Questions}

The main research question is substantially to investigate the causes of the poor access to palliative care regarding the availability of opioids. Based on the findings of the investigation, a novel model should be built to tackle the causes. 
Medical Technologies Journal, Volume: 3, Issue: 3, July-September 2019, Pages: 430-442. Doi :

https://doi.org/10.26415/2572-004X-vol3iss3p430-442

This section aims to figure out the novelty of the proposed model in the context of palliative care in Burkina Faso (BF) and the contribution to the literature.

Four research questions are presented in the introduction. Mainly, this editorial paper aims to investigate to what extent ICT can assist to improve the palliative care in BF, whereby to secure prescription against any forgery in order to prevent health safety issues. A further question is how ICT can underly the reporting process.

Most questions are already answered in the literature. Though, the answers need to be adapted to the context. Therefore, the context related questions are still open and are judicious to be posed.

\subsection{Theory of Changes and related Risks}

Two major assumptions were made regarding the improvement objectives. The health informatics literature widely presents cases of IT solutions that assist to improve care delivery and processes. Though, regarding the context (BF a financial and technological resource-poor country), the $\mathrm{H} 1$ can become challenging. Therefore, in situ-tests need to figure out.

$\mathrm{H} 2$ assumes that EMR can contribute to reducing overprescription. Theoretically, when the MD is aware of the medication history of a patient, he can adjust his prescription. Though an in-situ test is needed here too.

\subsection{Evidence Gathering}

At this stage of the study, the long-term and longitudinal test is in preparation to be conducted in the entire country to collect an important amount of data for evidence. The literature review and studies conducted prior have shown the potential of information systems to improve the health care delivery process and health outcomes.

\subsection{Contribution Assessment}

IoT is facing huge roadblocks such as automation, interoperability, and security issues such as data privacy. Using blockchain for IoT could solve some of these issues. However, Blockchain in its fundamental transparent. The novelty of this work is to combine FHIR and blockchain for IoT. FHIR on the blockchain will assume the data interoperability among the system and the IoT part will assist to autonomously and automatically collect data and secure them. In [23], a concept called FHIRChain is design and implemented. The evidence of the FHIRChain architecture has shown promise to fulfill the requirement of the Office of the National Coordinator for Health Information Technology (ONC). ONC requires all hospitals and clinics based in the USA to adhere to a secure and scalable data sharing to enable collaborative decision making. The objective of this requirement is to support interoperable medical data sharing. Based on the test conducted in the study reported in [23], and a prior study conducted on IoT in medical Emergency, the proposed model has the potential to produce accurate data, which can secure the opioid distribution, improve access to opioids in the entire country since the opioid order and supply chain will rely on accurate data on the real needs of the population. Paper-based reports would 
be replaced by digital report form, which gets automatically filled using the IoT technology without any manual implication.

\subsection{Additional Evidence}

This paper presents a Blockchain combined with FHIR on IoT Technology to improve access to opioids in Burkina Faso. Improving access to opioids implies to improve the supply and distribution chains, prevent opioid abuse and addiction by monitoring the prescription in order to prevent overprescription, hinder prescription fraud. It is also important to monitor and enact off-label prescription and use regulation.

Though the design and implementation and although, that blockchain on FHIR has shown in the USA, additional evidence on the blockchain on IoT technology using FHIR for data interoperability is needed to be collected in the context of palliative care in BF in order to show the potential and feasibility of such a technological and financial resource-poor health care system. The additional evidence collection is pursuing the objective to conduct an action study and thus adjust the designed model till to find out the suitable model parameters.

\subsection{Revision of the Contribution}

The revision of the contribution will be performed afterward the evidence gathering in the scope of an action study.

\section{Conclusion}

This study presents the opioids crisis and abuse. It focuses on Burkina Faso and analyzes the different factors contributing to the opioid shortage, challenges, and issues to adequately monitoring the opioid consumption, the supply chain, as well as the order aboard. Depth analysis of the directives released by $\mathrm{MoH}$ has revealed the limitation of these directives. A contextualized model proposed has the potential to overcome the different issues, though it is limited to stem the illegal distribution channel, which is the black market.

\section{Future Work}

The implemented and test of the proposed model will be reported in the future paper.

\section{Conflict of Interest Statement}

We certify that there is no conflict of interest with any financial organization in the subject matter or materials discussed in this manuscript.

\section{Authors' Biography}


Medical Technologies Journal, Volume: 3, Issue: 3, July-September 2019, Pages: 430-442. Doi :

https://doi.org/10.26415/2572-004X-vol3iss3p430-442

Dr. Thierry Oscar Edoh is an associate and affiliated researcher at the University of Bonn (Germany)/ Department of Pharmacy, visiting associate lecturer at the Institute of Mathematics and Physics (IMSP)/University Abomey-Calavi, (Benin-Africa), visiting lecturer at IUT Lokossa (Benin-Africa), and an affiliated researcher at the Technical University of Munich/department of Applied Software Engineering (Germany). He is a guest lecturer at many African, Asian, and East European Universities. He received his Diploma in computer sciences from the Technical University of Munich in Germany and held a Ph.D. at the German Federal Army University, where he worked for several years on the improvement of rural health care provision and access to healthcare in developing countries using ITC systems.

He performed postdoctoral research at the University of Bonn (Germany)/department of pharmacy. He worked on Drug Regulatory Affairs with a focus on Knowledge discovery and Drug marketing authorization. He is a member of IEEE.

Dr. Martin Lankoandé is anesthesia and Intensive Care physician, at the teaching Hospital Yalgado Ouédroago, in Burkina Faso. He received a Diploma in medicine from the University of Ouagadougou in Burkina Faso and holds a master's in anesthesiology from the Catholic University of Louvain in Belgium. He was award-winner of the first prize for excellence at the Burkina Faso National Secondary Education Olympiad Competition in 2004, the best prize of palliative care diffusion at the French-speaking international palliative care federations in Paris in June 2019. His research interests are anesthesia, intensive care, pain and palliative care, health informatics. He has authored about 30 original papers.

\section{9. $\quad$ References}

[1] T. O. Edoh and G. Teege, "Using information technology for an improved pharmaceutical care delivery in developing countries. Study case: Benin," J. Med. Syst., vol. 35, no. 5, pp. 1123-1134, 2011. https://doi.org/10.1007/s10916-011-9717-y PMid:21519942

[2] L. J. Paulozzi, K. A. Mack, and J. M. Hockenberry, "Variation among states in prescribing of opioid pain relievers and benzodiazepines - United States, 2012," J. Safety Res., vol. 51, pp. 125-129, 2014. https://doi.org/10.1016/j.jsr.2014.09.001

PMid:25453186

[3] M. M. Queiroz and S. Fosso Wamba, "Blockchain adoption challenges in supply chain: An empirical investigation of the main drivers in India and the USA," Int. J. Inf. Manage., vol. 46, no. September 2018, pp. 7082, 2019. https://doi.org/10.1016/j.ijinfomgt.2018.11.021

[4] R. Casado-Vara, J. Prieto, F. De La Prieta, and J. M. Corchado, "How blockchain improves the supply chain: Case study alimentary supply chain," Procedia Comput. Sci., vol. 134, pp. 393-398, 2018. https://doi.org/10.1016/j.procs.2018.07.193

[5] J. Richard and M. M. Reidenberg, "The risk of disciplinary action by state medical boards against physicians prescribing opioids," J. Pain Symptom Manage., vol. 29, no. 2, pp. 206-212, 2005.

https://doi.org/10.1016/j.jpainsymman.2004.05.009

PMid:15733811

[6] T. O. C. Edoh, A. Atchome, B. R. U. . Alahassa, and P. Pawar, "Evaluation of a Multi-Tier Heterogeneous Sensor Network for Patient Monitoring - The Case of Benin," in MMHealth '16 Proceedings of the 2016 ACM Workshop on Multimedia for Personal Health and Health Care, 2016, pp. 23-29. https://doi.org/10.1145/2985766.2985772

[7] T. O. Edoh, P. A. Pawar, B. Brügge, and G. Teege, "A Multidisciplinary Remote Healthcare Delivery System to Increase Health Care Access, Pathology Screening, and Treatment in Developing Countries," in Health Care Delivery and Clinical Science, IGI Global, 2017, pp. 269-302. https://doi.org/10.4018/978-1-5225-39261.ch013

[8] T. O. Edoh, Advanced Systems for Improved Public Healthcare and Disease Prevention. USA: IGI Global, Medical Information Science Reference (an imprint of IGI Global), 2018. 
Medical Technologies Journal, Volume: 3, Issue: 3, July-September 2019, Pages: 430-442. Doi : https://doi.org/10.26415/2572-004X-vol3iss3p430-442

[9] A. KHELASSI, V. V. Estrela, A. C. B. Monteiro, R. P. França, Y. Iano, and N. Razmjooy, "Health

4. 0: Applications, Management, Technologies and Review," Med. Technol. J., vol. 2, no. 4, pp. 262-276, Jan. 2019.

https://doi.org/10.26415/2572-004X-vol2iss4p262-276

[10] Léné SEBGO, "Manuel de gestion des déchets Médicaux," 2014.

[11] S. Bouda, "Manuel De Gestion Des Impacts," Ougadougou, 2010.

[12] D. Valdeolmillos, Y. Mezquita, A. González-Briones, J. Prieto, and J. M. Corchado, "Blockchain Technology: A Review of the Current Challenges of Cryptocurrency," in Blockchain and Applications, BLOCKCHAIN., vol. 1010, C. J. Prieto J., Das A., Ferretti S., Pinto A., Ed. 2020, pp. 153-160. https://doi.org/10.1007/978-3-030-23813$1 \_19$

[13] M. Mettler, "Blockchain technology in healthcare: The revolution starts here," 2016 IEEE 18th Int. Conf. eHealth Networking, Appl. Serv. Heal. 2016, pp. 16-18, 2016. https://doi.org/10.1109/HealthCom.2016.7749510

[14] S. Saberi, M. Kouhizadeh, J. Sarkis, and L. Shen, "Blockchain technology and its relationships to sustainable supply chain management," Int. J. Prod. Res., vol. 57, no. 7, pp. 2117-2135, 2019. https://doi.org/10.1080/00207543.2018.1533261

[15] H. Min, "Blockchain technology for enhancing supply chain resilience," Bus. Horiz., vol. 62, no. 1, pp. 35 45, 2019. https://doi.org/10.1016/j.bushor.2018.08.012

[16] S. M. Riazul Islam, Daehan Kwak, M. Humaun Kabir, M. Hossain, and Kyung-Sup Kwak, "The Internet of Things for Health Care: A Comprehensive Survey," IEEE Access, vol. 3, pp. 678-708, 2015. https://doi.org/10.1109/ACCESS.2015.2437951

[17] F. Hu, D. Xie, and S. Shen, "On the application of the internet of things in the field of medical and health care," Xplore IEEE - 2013 IEEE Int. Conf. Green Comput. Commun. IEEE Internet Things IEEE Cyber, Phys. Soc. Comput. GreenCom-iThings-CPSCom 2013, pp. 2053-2058, 2013.

[18] V. V Estrela, J. Hemanth, O. Saotome, E. G. H. Grata, and D. R. F. Izario, "Emergency Response CyberPhysical System for Flood Prevention with Sustainable Electronics," in Proceedings of the 3rd Brazilian Technology Symposium, Cham: Springer International Publishing, 2019, pp. 319-328. https://doi.org/10.1007/978-3-31993112-8_33

[19] T. O. Edoh and G. T. Pravin Amrut Pawar, Bernd Brügge, "A Multidisciplinary Remote Healthcare Delivery System to Increase Health Care Access , Pathology Screening, and Treatment in Developing

Countries :," Int. J. Healthc. Inf. Syst. Informatics, vol. 11, no. 4, pp. 1-31, 2016. https://doi.org/10.4018/IJHISI.2016100101

[20] Y. Chen, S. Ding, Z. Xu, H. Zheng, and S. Yang, "Blockchain-Based Medical Records Secure Storage and Medical Service Framework," J. Med. Syst., vol. 43, no. 1, 2018. https://doi.org/10.1007/s10916-018-1121-4 PMid:30467604

[21] A. Ekblaw, A. Azaria, J. D. Halamka, and A. Lippman, "A case study for blockchain in healthcare: 'MedRec' prototype for electronic health records and medical research data," OBD 2016 2nd Int. Conf. Open Big Data, vol. 13, p. 13, 2016.

[22] R. Gazzotti, C. Faron-Zucker, F. Gandon, V. Lacroix-Hugues, and D. Darmon, "Injecting Domain Knowledge in Electronic Medical Records to Improve Hospitalization Prediction," The Semant., vol. 1, H. P. et Al., Ed. Springer, Cham, 2019, pp. 116-130. https://doi.org/10.1007/978-3-030-21348-0_8

[23] P. Zhang, J. White, D. C. Schmidt, G. Lenz, and S. T. Rosenbloom, "FHIRChain: Applying Blockchain to Securely and Scalably Share Clinical Data," Comput. Struct. Biotechnol. J., vol. 16, pp. 267-278, 2018. https://doi.org/10.1016/j.csbj.2018.07.004 PMid:30108685 PMCid:PMC6082774

[24] Martin Lankoandé. Policy brief : Public and political awareness of palliative and elderly care in Burkina Faso. URL:

https://hospicecare.com/uploads/2018/6/Burkina\%20Faso\%20Presentation\%20Martin\%20Lankoande. pdf (Last time visited: November 2019) 